\title{
In-Situ Investigation of Local Boundary Migration During Recrystallization
}

\author{
YUBIN ZHANG, ANDY GODFREY, and DORTE JUUL JENSEN
}

A combination of electron channeling contrast (ECC) and electron backscatter diffraction pattern (EBSP) techniques has been used to follow in situ the migration during annealing at $323 \mathrm{~K}\left(50{ }^{\circ} \mathrm{C}\right)$ of a recrystallizing boundary through the deformed matrix of high-purity aluminum rolled to 86 pct reduction in thickness. The combination of ECC and EBSP techniques allows both detailed measurements of crystallographic orientations to be made, as well as tracking of the boundary migration with good temporal resolution. The measured boundary velocity and the local boundary morphology are analyzed based on calculations of local values for the stored energy of deformation. It is found that the migration of the investigated boundary is very complex with significant spatial and temporal variations in its movement, which cannot directly be explained by the variations in stored energies, but that these variations relate closely to local variations within the deformed microstructure ahead of the boundary, and are found related to the local spatial arrangements and misorientations of the dislocation boundaries. The results of the investigation suggest that local analysis, on the micrometer length scale, is necessary for the further understanding of recrystallization boundary migration mechanisms.

DOI: $10.1007 / \mathrm{s} 11661-014-2222-4$

(C) The Author(s) 2014. This article is published with open access at Springerlink.com

\section{INTRODUCTION}

DURING recrystallization of deformed metals, new nuclei form and grow by boundary migration through the deformed matrix. The velocity, $v$, of a migrating boundary in response to a driving force, $F$, is generally expressed by Eq. [1]:

$$
v=M F,
$$

where $M$ is the mobility of the boundary. ${ }^{[1]}$ For recrystallization, $F$ is generally considered as the stored energy in the deformed matrix. Many studies have shown that deformation microstructures in most metals are highly heterogeneous ${ }^{[2-4]}$ and that they depend on the crystallographic orientations of the grains present. ${ }^{[5-8]}$ Consequently, $F$ may vary significantly on the local scale. Moreover, the mobility, $M$, depends on many parameters, including the misorientation of the boundary, ${ }^{[9-11]}$ the boundary plane,${ }^{[12,13]}$ and the magnitude of the driving force. ${ }^{[14,15]}$ Therefore, despite the simple relationship expressed in Eq. [1], the heterogeneous nature of deformed microstructures, and hence the wide range of values of $F$ and $M$, suggests that in reality the

YUBIN ZHANG, Researcher, and DORTE JUUL JENSEN, Professor, are with the Danish-Chinese Center for Nanometals, Section for Materials Science and Advanced Characterization, Department of Wind Energy, Technical University of Denmark, Ris Campus, 4000 Roskilde, Denmark. Contact e-mail: yubz@dtu.dk ANDY GODFREY, Professor, is with the Key Laboratory of Advanced Materials (MOE), School of Materials Science and Engineering, Tsinghua University, Beijing 100084, P.R. China.

Manuscript submitted September 3, 2013.

Article published online February 20, 2014 migration of recrystallization boundaries is complex, such that the migration possibilities of a recrystallization boundary may vary significantly in space and time. For example, in-situ three-dimensional X-ray diffraction (3DXRD) observations of the growth of a grain during recrystallization have shown that the migration of recrystallization boundaries, even in weakly deformed single crystals, is quite inhomogeneous. ${ }^{[16,17]}$ These measurements have revealed that the migration of individual boundary segments occurs in a jerky stopgo fashion, and that locally fairly large protrusions/ retrusions (i.e., where locally some parts of a boundary segment migrate further/less than the neighboring parts) form and evolve on many boundaries. ${ }^{[16]}$ Recently, ex-situ electron backscatter diffraction pattern (EBSP) investigations have shown that even neighboring boundary segments, with very similar misorientations to the nearby deformed microstructure and with similar driving force $(F)$ from the deformed matrix, can behave quite differently. ${ }^{[18]}$

Subsequent to the publication of the 3DXRD results, theoretical models, aiming at understanding the local boundary migration in terms of the formation of pro- $/$ re-trusions, have been suggested by Godiksen et al., ${ }^{\text {19,20] }}$ Martorano et al., ${ }^{[21,22]}$ and Moelans et al. ${ }^{[23]}$ None of these models are, however, yet able to reproduce and predict typical experimental observations of boundary migration. Experimental ex-situ and in-situ electron channeling contrast (ECC) studies on the migration of recrystallizing boundaries have shown that both the deformed microstructure and the formation of pro-/ re-trusions can affect the local boundary migration. ${ }^{24,25]}$ However, key information, such as the local stored energy in the deformed matrix ahead of the migrating 
boundary, and the misorientation angles across the migrating boundary, cannot be obtained from ECC images.

The aim of the present work is, therefore, to study the migration of a recrystallizing boundary by in-situ annealing using both the ECC and the EBSP techniques. The benefit of this combination is that the EBSP maps contain crystallographic information with a sufficient angular resolution to be used for calculation of the local stored energy, while the ECC images provide both a higher spatial resolution as well as faster data acquisition than the EBSP maps. During annealing, the boundary migration can, therefore, be tracked over a large area using ECC images with a relatively high time resolution ( $\sim 1.5$ minutes), which cannot be achieved by EBSP investigations. Additionally, by following the trace of the migrating boundary as seen in the ECC images, and comparing this with the characteristics of the deformed microstructures characterized by EBSP in the same area before annealing, the migration of the recrystallizing boundary can directly be related to the deformed microstructure. The data thus allow a detailed analysis of the effects of local variations in the deformed microstructure on the boundary migration, as well as an evaluation of Eq. [1] on the local scale.

\section{EXPERIMENTAL}

The material used for this investigation was $99.996 \mathrm{pct}$ pure aluminum, annealed at $823 \mathrm{~K}\left(550{ }^{\circ} \mathrm{C}\right)$ for 24 hours to obtain an initial grain size of several millimeters. This initial material was cold rolled to 50 pet reduction in thickness at room temperature (RT) and subsequently rolled further to a total reduction of 86 pet in thickness at liquid-nitrogen temperature to avoid dynamic recrystallization during rolling. After rolling, the material was stored in a freezer at $255 \mathrm{~K}$ $\left(-18{ }^{\circ} \mathrm{C}\right)$.

When the material was examined the first time after storage, it was found to be already partially recrystallized. The recrystallization may have taken place any time during the post-deformation handling, for example, during cutting or polishing. However, for the purposes of the current investigation the fact that the material had already partly recrystallized during preparation does not in any way affect the analysis undertaken, as we relate the migration of the recrystallization boundary directly to the characteristics of the local deformation microstructure ahead of the boundary without assuming how the microstructure looked just after deformation (which is also irrelevant for an analysis of a recrystallizing boundary at a given annealing stage). For simplicity, however, the non-recrystallized parts of the microstructure are in the following referred to as the "deformed microstructure," although clearly some recovery has taken place in the unrecrystallized parts of the sample.

The in-situ experiment was carried out using a heating stage (DEBEN UK Ltd.) with a temperature range from $248 \mathrm{~K}$ to $323 \mathrm{~K}\left(-25^{\circ} \mathrm{C}\right.$ to $\left.50{ }^{\circ} \mathrm{C}\right)$, in a Zeiss Supra 35 thermal field emission gun scanning electron microscope (SEM). The sample of size $\sim 2 \times 2 \times 6 \mathrm{~mm}^{3}$ was first cooled from $\mathrm{RT}$ to $273 \mathrm{~K}\left(0{ }^{\circ} \mathrm{C}\right)$ inside the $\mathrm{SEM}$ to stabilize the microstructure for the initial ECC examination and EBSP mapping of a selected area containing both deformed matrix and a recrystallizing grain separated by a long boundary (see Figures 1(a) and (b)). For the EBSP measurements, an electron beam step size of $0.25 \mu \mathrm{m}$ was used.

The sample was then heated to $323 \mathrm{~K}\left(50{ }^{\circ} \mathrm{C}\right)$ and the migration of the recrystallizing boundary was recorded using the ECC technique at intervals of 100 seconds. In total 86 frames were recorded (the full set of the ECC images can be found in the supplementary materials). From the full set of ECC images the boundary position was determined at every time step (100 seconds interval), and these observations are used to prepare a sketch of tracings showing the recrystallization boundary position (see Figure 1(c)). A detailed description focusing on the observation of surface grooving which is observed in some places can be found in Reference 26. No notable grooving is, however, observed in the area
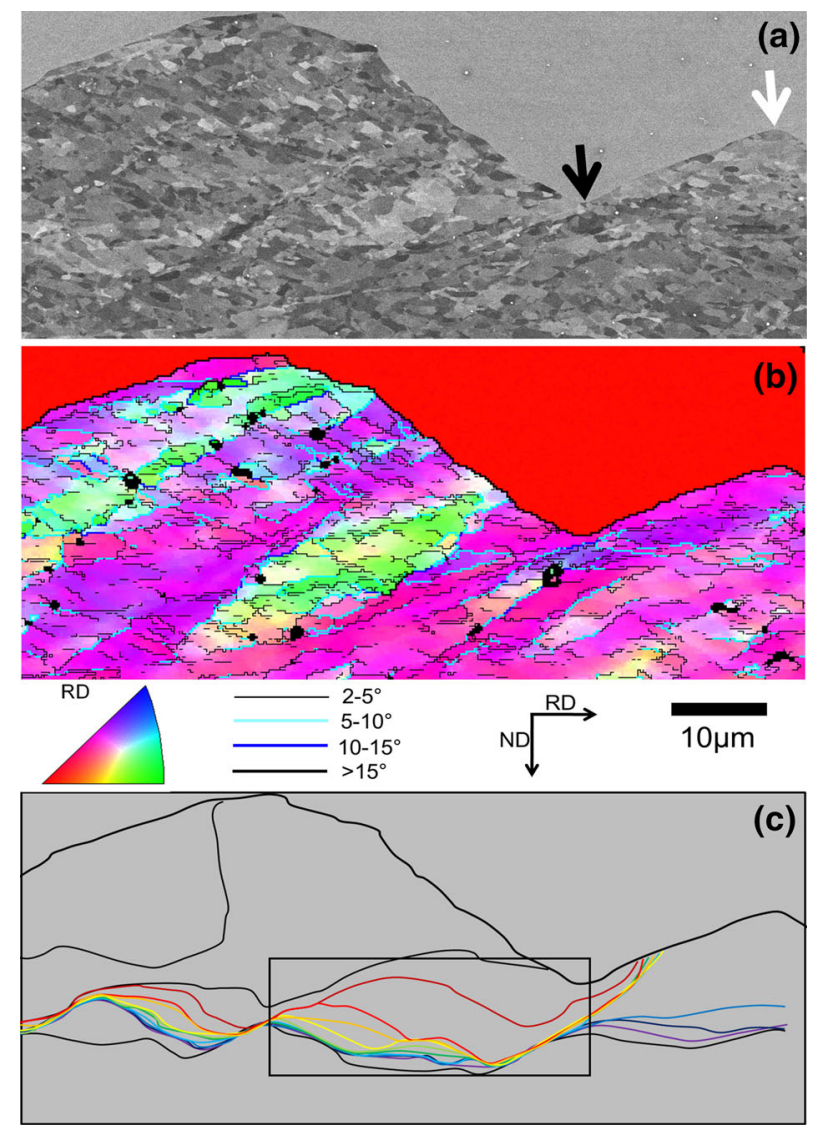

Fig. 1-ECC image (a) and EBSP map (b) showing the microstructure of the initial partially recrystallized sample containing a recrystallizing grain and deformed matrix. Black and white arrows point out a large protrusion and retrusion, respectively. In the EBSP map, the colors represent the crystallographic direction parallel to the rolling direction. Thin black lines, thick aqua, blue, and black lines are used to represent boundaries with misorientations $>2,>5,>10$, and $>15$ deg, respectively. Black pixels are non-indexed. (c) Traces of the position of the recrystallization boundary during the whole annealing duration, where for clarity only the position at every fifth time step (500 s interval) is shown. A rectangle marks the area in which the boundary migration is analyzed in the present work. 

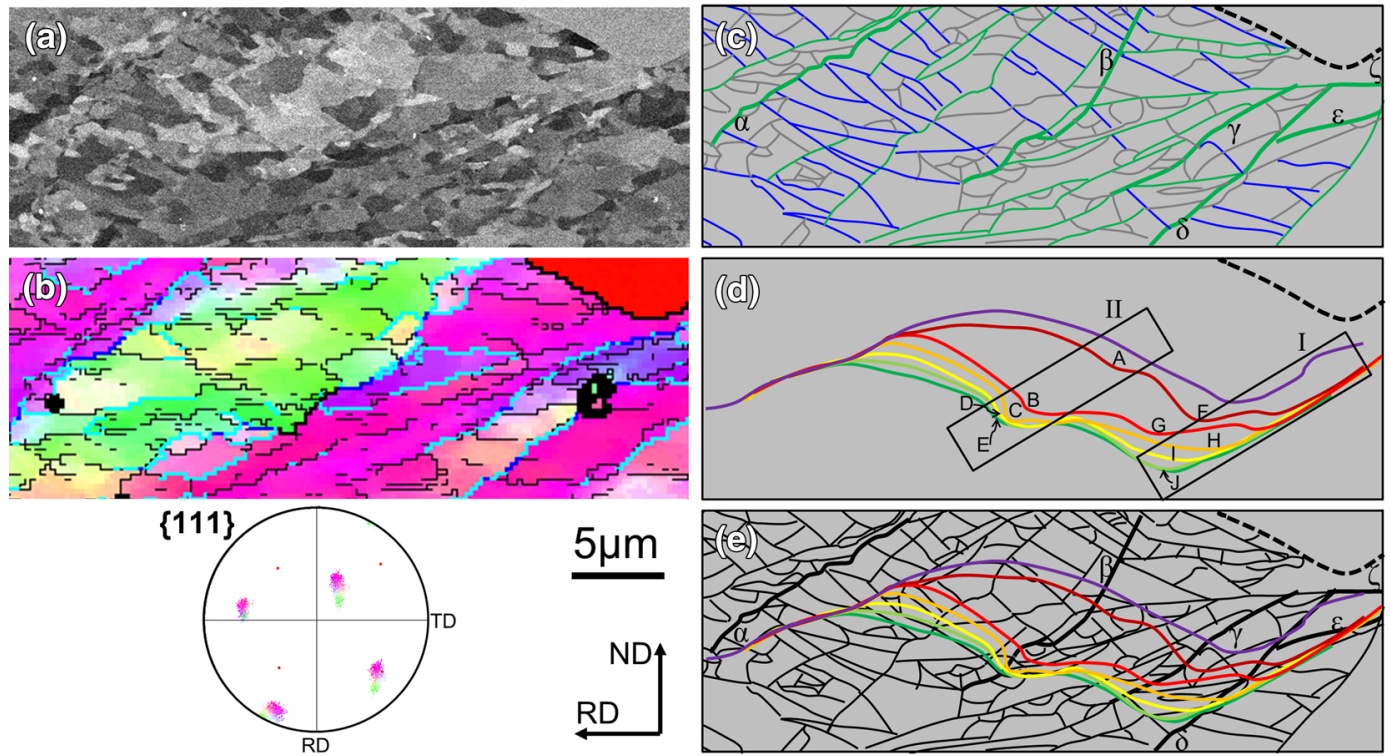

Fig. 2-(a) ECC image showing the microstructure in the area marked in Fig. 1. A recrystallizing grain is seen in the top right corner. (b) EBSP map showing the same microstructure as shown in (a), together with its $\{111\}$ pole figure. The color coding for (b) is the same as that in Fig. 1(b). (c) Sketch of the dislocation boundaries in the deformed matrix based on the ECC image in (a). Microbands and LGBs are shown by blue and green lines, respectively. Dislocation cells are shown by dark gray lines. (d) Traces of the position of the recrystallizing boundary during the first seven $100 \mathrm{~s}$ time steps. Box I and II mark local areas where detailed analysis is carried out. Letters A to J are used to identify protrusions. (e) Non-colored image of the deformed microstructure (from (c)) superimposed with recrystallizing boundary traces shown color. In (c) and (e) boundaries containing segments with misorientation $>10$ deg are highlighted by thicker lines and marked by $\alpha-\zeta$. The initial recrystallizing boundary position is shown by a dashed black line in (c) to (e).

marked by a rectangle in Figure 1(c) and in nearby regions. The boundary migration in this area is investigated in detail and related to the deformed microstructure in the present work.

\section{RESULTS}

The ECC image and EBSP map of the selected area (marked by a rectangle in Figure 1(c)) are shown in Figures 2(a) and (b). The orientation of the recrystallized grain is $\sim 12 \mathrm{deg}$ from the ideal Cube orientation, $\{100\}\langle 001\rangle$, while the average orientation of the deformed matrix calculated from the EBSP map is rotated about $20 \mathrm{deg}$ from the $\mathrm{S},\{123\}\langle 634\rangle$, orientation. It can be seen from the ECC image that the deformation microstructure is heterogeneous, and is subdivided by two sets of extended dislocation boundaries. One set is narrowly spaced with dislocation boundaries lying at angles of -30 to -35 deg to $\mathrm{RD}$, forming a microband (MB) structure. ${ }^{[27]}$ The other set is more irregularly spaced, lying over a wider range of angles from 15 to 50 deg to RD. In some places the second more irregularly spaced set has widened to form localized glide bands (LGBs). ${ }^{[28]}$ A sketch of the deformation microstructure corresponding to the ECC image is shown in Figure 2(c), where the two sets of dislocation boundaries are shown in blue and green, respectively. In the sketch the remaining boundaries, which are interconnecting boundaries, are shown in dark gray. Dislocation boundaries containing segments with misorientations $>10$ deg in the EBSP map (as marked by $\alpha-\zeta$ in Figure 2(b)) are highlighted by thicker lines in the sketch. It can be seen that all these boundaries are part of LGBs and thus are shown in green in Figure 2(c). The deformed microstructure resembles that seen in a S-orientation single crystal after channeldie deformation to a medium-high strain, ${ }^{[28,29]}$ though the scale is coarser due to the occurrence of some recovery.

The traces of the recrystallizing boundary position in this area over the first seven 100 seconds time steps are sketched in Figure 2(d). After the first time step the recrystallizing boundary, shown by the purple line in Figure 2(d), consists of a large retrusion and protrusion, and most of the boundary segments are parallel to either the MBs or one of the LGBs as marked by $\alpha-\delta$ in Figure 2(c). After the second time step the two ends of the boundary remain fixed, where long, flat boundary segments develop, which do not migrate further during the entire duration of the investigated annealing experiment. In between these fixed points, the migration of the recrystallizing boundary is quite heterogeneous through all the observed time steps: many small pro-/ re-trusions form during the migration, and the migration velocity varies in space and time, with fast migration observed in particular during time interval \#2 (i.e., between the second and third time steps, see Figure 2(d)).

The migration velocity, $v$, of the interface can be calculated using Eq. [2]:

$$
v=A / l t,
$$

where $A$ is the area through which a migrating boundary of a length, $l$, migrates over a given annealing time interval, $t$. The velocity can also be measured by a 
line-intersect method, i.e., by drawing a line across the migrating boundary and measuring the migration distance during each time interval. In the present work, we used Eq. [2] to calculate the average migration velocity of the boundary during each time interval, and used a line-intersect method (described below) to estimate the local spread in the boundary velocity. For determination of the local boundary velocity using the line-intersect method, a series of lines were drawn across the whole boundary length, with spacing of $0.5 \mu \mathrm{m}$, and parallel to the long side of the box I in Figure 2(d). This line direction was chosen as it corresponds to the overall (average) boundary migration observed macroscopically over the studied boundary length.

Figure 3 shows for each time interval the average migration velocity, calculated over the entire investigated length of the migrating boundary, against the stored energy in the deformed matrix. The stored energy in the deformed matrix consumed during migration of the boundary between neighboring traces was calculated based on the EBSP data using methods described in Reference 30 . In the calculation, all dislocation boundaries with misorientation $>1.5 \mathrm{deg}$ were taken into account and the boundary energy was calculated based on Read-Shockley equation with maximum boundary energy of $\sigma_{\max }=0.324 \mathrm{~J} / \mathrm{m}^{2[24]}$ for boundaries with misorientation $>15 \mathrm{deg}$. A best line fit, weighted by the standard deviation in calculated velocity, to the data is shown in Figure 3, however, it is evident that this does not fit the data well.

One reason for deviations from a single straight line in Figure 3 could be a variation of the mobility with time, as the boundary continually meets new parts of the deformed matrix during migration. The misorientations between the recrystallizing grain and the deformed matrix in the consumed area at each time interval have, therefore, been calculated, and are shown in Figure 4. Except for a slight trend for the rotation axes to rotate from [ $\left.\begin{array}{lll}3 & 3 & 2\end{array}\right]$ toward [ 1110$]$ with increasing annealing time, the variation in misorientations over the observed annealing time is only small, and may not explain the

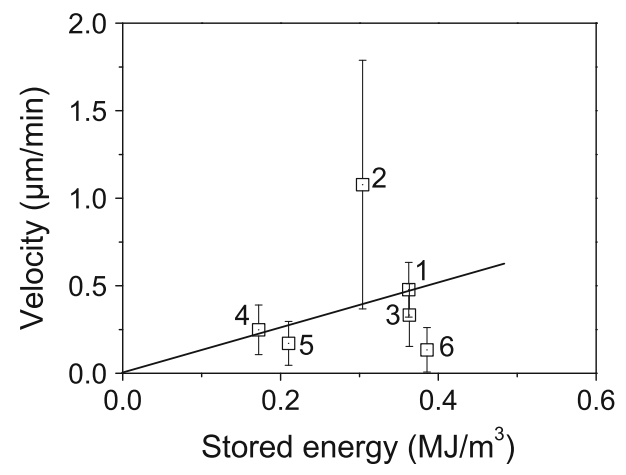

Fig. 3-Migration velocity measured using Eq. [2] as a function of stored energy in the deformed matrix for each time interval averaged over the whole boundary length studied. Numbers show the time intervals. Error bars show standard deviations calculated based on the velocities, which are measured by a line-intersect method. Solid line represents a best fit, weighted by the standard deviation in calculated velocity, to the data using Eq. [1]. large scatter in Figure 3. In particular, the fast boundary movement in time interval \#2 and the slow movement in interval \#6 cannot be attributed to big changes in the misorientation distributions.

\section{DISCUSSION}

The migration of boundaries during recrystallization is generally described based on an overall characterization of a given sample, by measuring the stored energy over the whole sample and by averaging the boundary velocity over many recrystallizing boundaries. ${ }^{[31,32]}$ In the present work we have analyzed the local boundary migration following in situ the motion of a single boundary segment and found that there are large variations in the boundary velocity, which represent deviations from a straight line relationship as described in Eq. [1] (see Figure 3), and which cannot be explained by changes in the misorientation characteristics during boundary migration.

One may speculate whether the variation in Figure 3 may relate to inhomogeneity of the deformed microstructure, as variations exist even within the relatively small area investigated in this study. Indeed it is observed that part of the recrystallizing boundary in box I (Figure 2(d)) does not migrate from the second time step onwards, and that the boundary migration in this region can be related to the dislocation boundary $\delta$. Boundary segments within box I migrate quickly across the dislocation boundary $\delta$ in the time interval \#1 (from the purple line to the dark red line in Figures 2(d) and (e)) but remain fixed once the higher stored energy associated with the dislocation boundary $\delta$ has been consumed. Boundary segments adjacent to the part that remains fixed can, however, still migrate along the dislocation boundary $\delta$ driven by the higher stored energy, leading to formation of an even longer section of flat stationary boundary (see Figure 2(d)). This process leads to formation of protrusions $\mathrm{H}$ to $\mathrm{J}$ at the front end of the long flat boundary. These protrusions, referred to as sawtooth shaped because of their shape, ${ }^{[33]}$ are expected to maintain this shape as long as some length of the recrystallizing boundary remains flat, following a long dislocation boundary with high misorientations. In a similar way, a long, flat non-migrating boundary segment develops at the left side of Figure 2(d), associated in this case with dislocation boundary $\alpha$.

In between these flat stationary boundary segments, local boundary migration with relatively high velocity is generally accompanied by formation of protrusions, e.g., protrusions $\mathrm{A}$ to $\mathrm{G}$ (because of their shape they are classified as rounded protrusions ${ }^{[33]}$ ). These protrusions can be related to dislocation boundaries $\beta$ and $\gamma$ (see Figure 2(e)). To investigate the detailed relationships between the boundary shape and the dislocation boundaries in this area, the maximum curvature dragging force $\left(F_{\sigma, \max }\right)$ has been calculated for each of the protrusions, and the resulting data are plotted as a function of the local stored energy in Figure 5. The value of $F_{\sigma, \max }$ was calculated using equation, $F_{\sigma, \max }=2 \sigma / r_{\min }$, where $\sigma$ is the grain boundary energy and $r_{\min }$ is the minimum 


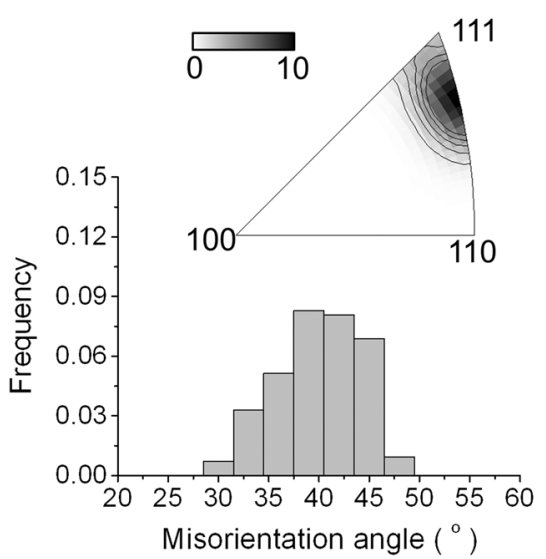

(a)

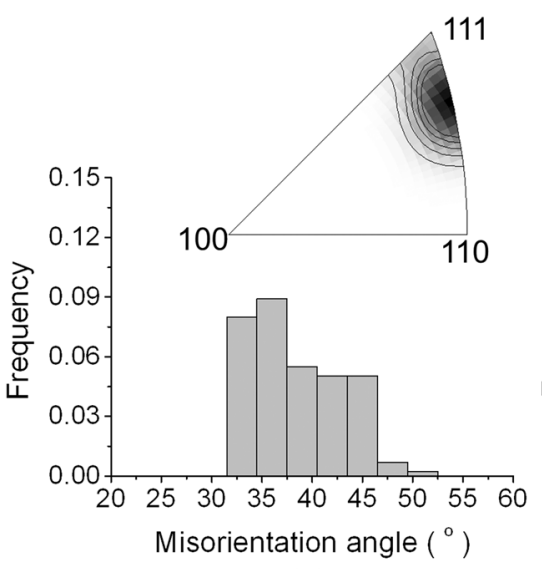

(b)

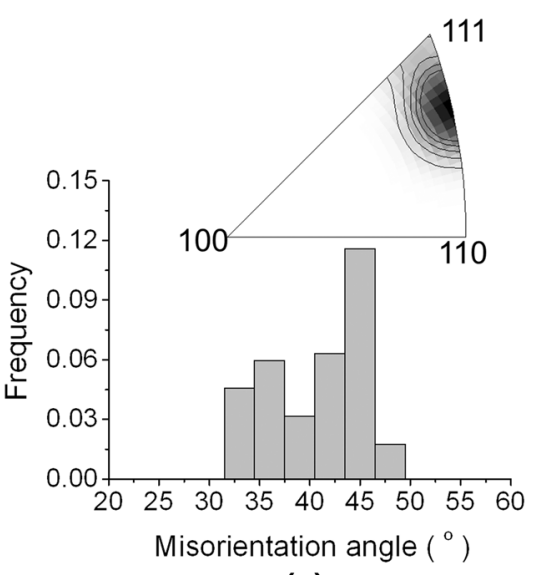

(c)

Fig. 4- (a) through (c) Misorientation distributions between the recrystallized grain and the consumed deformed matrix during time intervals \#2, $\# 4$, and \#6, respectively. The histograms show the distribution of misorientation angles, and the inverse pole figures (insets) show the distribution of the misorientation rotation axes.

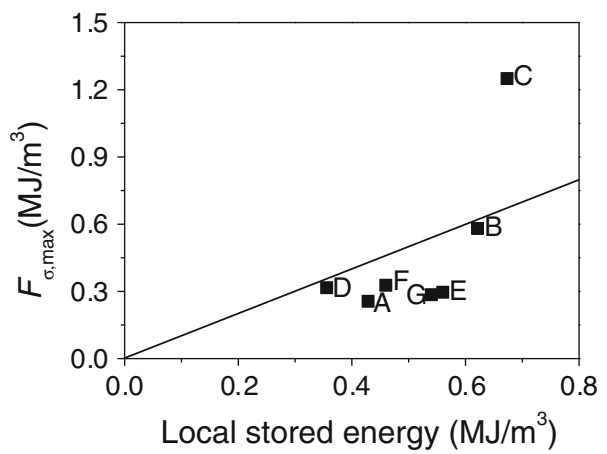

Fig. 5-Maximum curvature dragging forces $\left(F_{\sigma, \max }\right)$ from the rounded protrusions A to $G$ (marked in Fig. 2(d)) against the local stored energies calculated from the deformed microstructure consumed by the protrusions. The solid line represents the case for the local stored energy being equal to the dragging force, $F_{\sigma \text {,max }}$; protrusions with values below this line are, therefore, expected to move forward, and protrusions with values above this line are expected to stop migrating.

radius of the curvature of each protrusion. For calculation of $r_{\text {min }}$, each protrusion was fitted with a third order polynomial equation. A detailed description of the calculation procedure can be found in References $24,25,34$. The stored energy values are calculated based on EBSP data within the area under each protrusion, which can be considered as the driving force for the formation of the protrusions. Figure 5 indicates a general tendency that local high stored energy in the deformed microstructure leads to protrusions with large values of $F_{\sigma, \max }$.

The formation of pro-/re-trusions in turn has effects on the boundary migration. For example, for the local boundary migration in box II (see Figure 2(d)), the length of boundary covered by this box migrates with increasing speed from protrusion $\mathrm{A}$ to $\mathrm{B}$, driven by removal of dislocation boundary $\beta$, which has an increasing misorientation angle along its length (from $9 \mathrm{deg}$ at protrusion $\mathrm{A}$, to $14 \mathrm{deg}$ in between protrusions $\mathrm{A}$ and $\mathrm{B}$, to $18 \mathrm{deg}$ at protrusion $\mathrm{B}$ ). However, even though the dislocation boundary misorientation in front of protrusion $\mathrm{B}$ is still higher $(22 \mathrm{deg})$, the local boundary migration velocity decreases after reaching position $\mathrm{B}$, due to the increased dragging force at $\mathrm{B}$ from the increasing curvature at the tip of the protrusion. This combined effect of local driving and dragging forces on the local migration rates can also be seen in Figure 5. For example, the value of $F_{\sigma, \max }$ for protrusion $\mathrm{C}$ is significantly higher than the driving force from the local stored energy, i.e., the dragging force of the tip of protrusion $\mathrm{C}$ is much larger than the driving force, suggesting that $\mathrm{C}$ should remain fixed during the next time step, as in fact is observed (see Figure 2(d)). Similarly, the values of $F_{\sigma, \max }$ for protrusions B and D are almost equal to the driving force from the local stored energy, so the velocity of boundary segments at protrusions $\mathrm{B}$ and $\mathrm{D}$ is expected to decrease or fall to zero, which also agrees with the experimental results. The driving force from the local stored energy is higher than the values of $F_{\sigma, \max }$ for protrusions $\mathrm{A}, \mathrm{F}, \mathrm{G}$, implying that all these protrusions could move during next time step, which also agrees well with the experimental observation. Note that protrusion $\mathrm{E}$ cannot be evaluated because it is only observed to form the last time step.

Based on the importance of local pro-/re-trusions on boundary migration, it has been suggested that the expression for migration velocity, $v$, can be modified as $^{[24,34]}$ :

$$
v=M\left(F_{\mathrm{s}}+F_{\sigma}\right),
$$

where $F_{\mathrm{s}}$ is local driving forces from the stored energy in the deformed microstructure, and $F_{\sigma}$ is net dragging/ driving forces (calculated with a sign) from the curvature of the pro-/re-trusions, integrated over the length, $\Delta l$, of boundary segments within the area investigated, i.e., $F_{\sigma}=\int_{\Delta \mathrm{l}} F_{\sigma, i}$. Note $F_{\sigma}$ is generally smaller than $F_{\sigma, \max }$ in magnitude. Figure 6 plots the migration velocity as a function of the total local driving forces, $F=F_{\mathrm{s}}+F_{\sigma}$, for the boundary segments included in box II in Figure 2(d). Compared to Figure 3, it is evident that when the local migration within box II is 


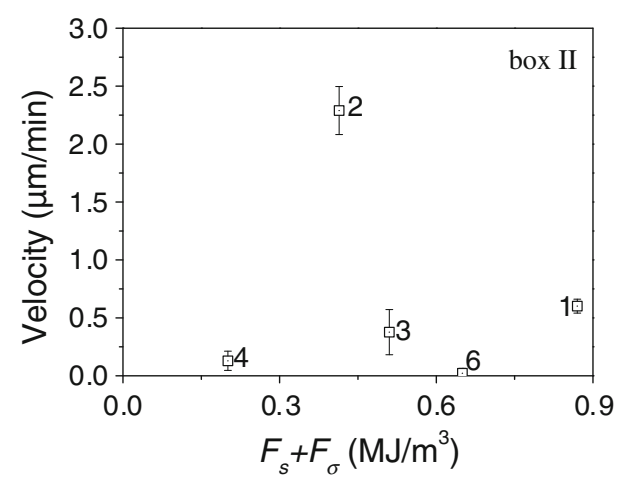

Fig. 6-Migration velocity of the recrystallizing boundary as a function of total driving force, $F=F_{\mathrm{s}}+F_{\sigma}$, for boundary segment in box II in Fig. 2(d) for each time interval. Numbers show the time intervals.

considered, instead of the migration of the whole boundary, the error bars (here given by the standard deviation on the measurements) are significantly reduced, which confirm the advantage of a local analysis of boundary migration compared to an analysis based on global (average) considerations. However, it should be noted that the deviation from a best fit straight line between velocity and net local driving force (not shown in the figure) is even larger than seen in Figure 3 (where only the force due to the local stored energy of deformation is considered). This large deviation may relate to several parameters including: (1) the average net curvature dragging/driving forces from pro-/ re-trusions, $F_{\sigma}$, is determined only based on the boundary traces at the start of each time interval; (2) the calculated stored energy and curvature of the protrusions are based on a 2D picture of the microstructure, which may imperfectly represent the complete 3D microstructure; (3) the mobility of individual boundary segments may vary with boundary plane, and in general the mobility may be more complex than hitherto considered, as discussed in Reference 12; and (4) the migration of individual recrystallization boundary segments depends in concert on the motion of neighboring (and beyond) segments (see, for example, a discussion on this as related to grain growth ${ }^{[35]}$. Of these four parameters, the first two are believed to be less important as (1) the variation in stored energy during migration in the local region over the short time intervals of 100 seconds is not expected to be large and (2) the protrusions often form ridges along TD (and are not sharp peaks) meaning that the curvature contribution from the third dimension is less important. Further clarification of the use of Eq. [3] to account for local migration behavior will, however, require detailed full 4D (i.e., $[x, y, z, t]$ ) experimental characterizations with a spatial, angular, and temporal resolutions that can resolve with sufficient accuracy the deformation microstructure and boundary migration. These requirements cannot be fully satisfied with existing experimental techniques, but work is underway which may ultimately lead to experiments of this type. Nevertheless, the present observations clearly demonstrate that the geometry and stored energy distribution within the deformation microstructure play a key role in the formation of pro-/re-trusions and in the migration of recrystallization grain boundaries.

\section{CONCLUSIONS}

A detailed study has been carried into boundary migration during recrystallization by making use of in-situ ECC observations of boundary movement coupled with initial EBSP measurements of the crystal orientations in the deformed matrix. The results of the investigation highlight the complexity of boundary migration during recrystallization. The following conclusions are obtained.

1. The migration of the recrystallizing boundary is very irregular in both space and time: the migration is sometimes held up, while fast at other times, and is accompanied by formation of sawtooth shaped and rounded pro-/re-trusions on the boundary.

2. The arrangement of dislocation boundaries in the deformed microstructure defines the local boundary migration such that qualitatively the migration is well understood.

3. Quantitative understanding of migration is more challenging, as a large deviation from a simple straight line relationship between the boundary velocity and local driving force is observed. It is suggested that such a deviation may be related mainly to uncertainties in knowledge about the appropriate value of mobility, $M$, which may be complex and may depend on many parameters, as well to the fact that individual boundary segments are strongly affected by the motion of neighboring segments. Both very advanced modeling and experimental tools are required to clarify these issues.

\section{ACKNOWLEDGMENTS}

The authors gratefully acknowledge the support from the Danish National Research Foundation (Grant No DNRF86-5) and the National Natural Science Foundation of China (Grant No. 51261130091) to the Danish-Chinese Center for Nanometals, within which this work has been performed.

\section{OPEN ACCESS}

This article is distributed under the terms of the Creative Commons Attribution License which permits any use, distribution, and reproduction in any medium, provided the original author(s) and the source are credited.

\section{ELECTRONIC SUPPLEMENTARY MATERIAL}

The online version of this article (doi: 10.1007/s11661-014-2222-4) contains supplementary material, which is available to authorized users. 


\section{REFERENCES}

1. F. Haessner: Recrystallization of metallic materials, Dr. Riederer Verlag GmbH, Stuttgart, 1978

2. N. Hansen and D. Juul Jensen: Mater. Sci. Technol., 2011, vol. 27, pp. $1229-40$.

3. A.S. Taylor, P. Cizek, and P.D. Hodgson: Acta Mater., 2012 vol. 60, pp. 1548-69.

4. F.J. Humphreys and P.S. Bate: Acta Mater., 2007, vol. 55 pp. 5630-45.

5. X. Huang and G. Winther: Philos. Mag., 2007, vol. 87, pp. 5189-5214.

6. Q. Liu, D. Juul Jensen, and N. Hansen: Acta Mater., 1998, vol. 46, pp. 5819-38.

7. A. Albou, J.H. Driver, and C. Maurice: Acta Mater., 2010, vol. 58, pp. 3022-34.

8. F.X. Lin, A. Godfrey, and G. Winther: Scripta Mater., 2009, vol. 61, pp. 237-40.

9. P.A. Beck, P.R. Sperry, and H. Hu: J. Appl. Phys., 1950, vol. 21, pp. 420-25.

10. G. Gottstein and L.S. Shvindlerman: Grain Boundary Migration in Metals: Thermodynamics, Kinetics, Applications, CRC Press Taylor \& Francis Group, Boca Raton, 2010.

11. M. Winning, A.D. Rollett, G. Gottstein, D.J. Srolovitz, A. Lim, and L.S. Shvindlerman: Philos. Mag., 2010, vol. 90, pp. 3107-28.

12. D.L. Olmsted, E.A. Holm, and S.M. Foiles: Acta Mater., 2009, vol. 57 , pp. 3704-13.

13. J. Hjelen, R. Ørsund, and E. Nes: Acta Metall. Mater., 1991, vol. 39, pp. 1377-1404.

14. D.L. Olmsted, S.M. Foiles, and E.A. Holm: Scripta Mater., 2007, vol. 57, pp. 1161-64.

15. E.A. Holm and S.M. Foiles: Science, 2010, vol. 328, pp. 1138-41.

16. S. Schmidt, S.F. Nielsen, C. Gundlach, L. Margulies, X. Huang, and D. Juul Jensen: Science, 2004, vol. 305, pp. 229-32.

17. S. Van Boxel, S. Schimdt, W. Ludwig, Y.B. Zhang, D. Juul Jensen, and W. Pantleon: Mater. Trans., 2014, vol. 55, pp. 128-36.

18. Y.B. Zhang, A. Godfrey, Q. Liu, W. Liu, and D. Juul Jensen: Acta Mater., 2009, vol. 57, pp. 2631-39.
19. R.B. Godiksen, Z.T. Trautt, M. Upmanyu, J. Schiøtz, D. Juul Jensen, and S. Schmidt: Acta Mater., 2007, vol. 55, pp. 6383-91.

20. R.B. Godiksen, S. Schmidt, and D. Juul Jensen: Model. Simul. Mater. Sci. Eng., 2008, vol. 16 (065002), pp. 1-20.

21. M.A. Martorano, H.R.Z. Sandim, M.A. Fortes, and A.F. Padliha: Scripta Mater., 2007, vol. 56, pp. 903-06.

22. M.A. Martorano, M.A. Fortes, and A.F. Padilha: Acta Mater., 2006, vol. 54, pp. 2769-76

23. N. Moelans, A. Godfrey, Y.B. Zhang, and D. Juul Jensen: Phys. Rev. B, 2013, vol. 88 (054103), pp. 1-10.

24. Y.B. Zhang, A. Godfrey, and D. Juul Jensen: Scripta Mater., 2011, vol. 64, pp. 331-34.

25. Y.B. Zhang, A. Godfrey and D. Juul Jensen: Proceeding 31st Risø International Symposium, 2010, vol. 31, pp. 497-503.

26. Y.B. Zhang, A. Godfrey, and D. Juul Jensen: Mater. Sci. Forum, 2013, vol. 753, pp. 117-20.

27. D. Hughes and N. Hansen: Metall. Trans., 1993, vol. 24A, pp. 2021-37.

28. A. Godfrey, D. Juul Jensen, and N. Hansen: Acta Mater., 2001, vol. 49, pp. 2429-40.

29. A. Godfrey, N. Hansen, and D. Juul Jensen: Metall. Mater. Trans. A, 2007, vol. 38A, pp. 2329-39.

30. A. Godfrey, W.Q. Cao, N. Hansen, and Q. Liu: Metall. Mater. Trans. A, 2005, vol. 36A, pp. 2371-78.

31. R.A. Vandermeer, D. Juul Jensen, and E. Woldt: Metall. Mater. Trans. A, 1997, vol. 28A, pp. 749-754.

32. E.A. Jagle and E.J. Mittemeijer: Metall. Mater. Trans. A, 2012 , vol. 43A, pp. 1117-31.

33. D. Juul Jensen, F.X. Lin, Y.B. Zhang, and Y.H. Zhang: Mater. Sci. Forum, 2013, vol. 753, pp. 37-41.

34. Y.B. Zhang, A. Godfrey, and D. Juul Jensen: Comput. Mater. Continua, 2009, vol. 14, pp. 197-207.

35. E. Holm, G. Rohrer, A. Rollett, S. Foiles, and M. Chandross: Presentation at TMS 142nd Annual Meeting \& Exhibition, March 5, San Antonio, TX, 2013. 